\title{
Success is a puzzle: Sorting out the pieces with metaphor analysis
}

\author{
Brian Zamarripa Roman and Jacquelyn J. Chini \\ Department of Physics, University of Central Florida, 4111 Libra Drive, Orlando, FL, 32816
}

\begin{abstract}
Women in physics continue to be an underrepresented group, in part due to negative stereotypes and adverse environments that result from a masculine perception of physics. To address this, we explore and highlight feminine perspectives of success in physics. We focus on success because the term is often used by researchers to frame academic achievements; however, not much work explores how different people conceptualize success. For this study we conducted semi-structured interviews of women at various stages of education and careers in physics. One interview question specifically elicited a participant-constructed metaphor of success in physics. The interview data was then examined with metaphor analysis for structural metaphors, which gives us insight into the characteristics of success that are salient to the individual. For example, with the metaphor of a caramel apple one participant highlighted the difficulty (through the apple's tartness) and overall satisfaction of physics (through the caramel's sweetness).
\end{abstract}

\section{INTRODUCTION}

Women are an underrepresented group in physics in part due to the common perception of physics as a masculine field [1]. Masculine perceptions can create a climate that results in detrimental experiences for women in physics spaces, such as stereotype threat, imposter syndrome, microagressions (e.g., not being included or recognized in the workplace) and overt sexism [2]. Microaggressions that women experience can preserve the masculine perception of physics. To increase the representation of women, we must support women in their success and address the masculine views of physics. Thus, we are investigating how women conceptualize success in physics.

One method for exploring people's conceptualizations is metaphor analysis [3]. Metaphor analysis is a process of examining metaphorical expressions and categorizing them into themes that give insight into the perspectives and understandings underlying a concept. Metaphor analysis has been used to explore concepts such as teaching, learning, reading and writing in various fields, including physics education research [4-6]. We take a similar approach to conceptualize women's views of success in physics. Previous work has emphasized the need to understand the perception of causal factors that play a role in attaining success [7]. Attributing success in a task to causal factors can have an impact on motivation to engage in future tasks.

In this study we investigate the underlying structure of success in physics by performing metaphor analysis on metaphors constructed by women in physics. We analyze the metaphors with our participants to interpret their metaphors and identify factors that can lead to success in physics.

\section{METHODOLOGY}

\section{A. Guiding Frameworks}

We use Feminist Standpoint Theory (FST) to guide this study, as it allows us to bring social and political power to the forefront and recognize the power imbalance between researcher and researched [8]. The lead researcher is a man looking into perspectives held by women, which may further the power imbalance and bias the study design. Following FST, we can mitigate some of the exploitative effects of the power imbalance by elevating the voices of the participants. Harding [8] reminds us that research has traditionally been intrinsically "colonial," in that the process tends to exploit the resources and knowledge of those being researched for the benefit of the researcher. In light of the potentially exploitative nature of research and the fact that metaphor analysis greatly depends on the interpretation of the articulated metaphors, we involved participants in the analysis, in-line with practices that go beyond traditional member-checking $[9,10]$.

Metaphors are central to this study since the structure of metaphors can give us insight into the structure of the participants' thoughts and actions [3]. A socio-cultural perspective of metaphors also allows us to recognize that metaphors are constructed by and with an individual's society and culture [4]. Looking at metaphors through this lens can provide insight into individuals' conceptualizations of success and insight into the conceptualizations that are common within the culture of physics.

We also explored the perceived causes participants attributed to success in physics. Weiner's [7] model suggests attribution of success influences both expectancy of success and emotions, which in turn guide motivation. He notes that in achievement-related contexts, individuals attribute their success to four dominant causes: ability (e.g., being good at basketball due to natural height), effort (e.g., passing a test due to a month of studying), task difficulty (e.g., winning a bowling match due to using bumpers) and luck (e.g., winning a bet due to good luck). The extent to which an individual has control over these causes, their stability and whether they are external or internal to a person can influence persistence. For example, attributing success to internal factors, like ability or effort, can increase self-esteem or self-worth. Thus, we investigate the specific causes of success in physics identified by women and properties of those causes. 


\section{B. Data collection}

Data was collected in the form of semi-structured interviews that were on average about 30 minutes long. The specific metaphor data we analyzed here came from one prompt in the interview protocol that requested the participants to complete the thought, "Success in physics is like..." with a metaphor or an analogy. The prompt was given halfway through the interview after discussing what success in general looks like to the participants. After the metaphor was provided, the interviewer requested an explanation of the metaphor and probed the boundaries of the metaphor by asking questions about extensions to the metaphor that were not initially mentioned.

\section{Participants}

Participants were recruited from a large, public university in the southeastern United States. We sought participation from women in physics at different stages of a physics career, from undergraduate students, graduate students, postdocs and faculty, and asked those receiving the recruitment email to share it with local women in careers related to physics. In total we interviewed eleven participants.

Due to length restrictions we focus on the responses of two individuals to compare perspectives without sacrificing attention to detail of individual participants. The two perspectives presented were selected for their emphasis on similar aspects of success in physics. We selected two students because students were more readily available to participate in the collaborative analysis, described below. Future work will address prevalent themes in the full sample.

Participants selected their own pseudonyms. Samaria is an undergraduate junior physics major. Renae is soon to be a third-year graduate student in the planetary science track of the physics $\mathrm{PhD}$ program.

\section{Analysis}

Following the methods of Paulson and Theado [11] we closely examined the participants' responses by breaking them down into individual metaphorical expressions. The metaphorical expressions were identified using source domain elements to describe features of the target domain. The target domain is the conceptual domain that the person is trying to understand or explain; in our study this is "success in physics." The source domain is the conceptual domain from which a person constructs metaphorical expressions (e.g., caramel apple as listed in the abstract). Each expression was then coded into categories similar to those that emerged in the work of Paulson and Armstrong [5]. Paulson and Armstrong [5] categorized metaphors as describing either a process or product. For example, "College reading is like a cramp" describes a product, and "College reading is like putting together a puzzle" describes a process. They also categorized a metaphor as conveying or not conveying overtly negative views of reading. An example of an overtly negative view is "Reading is a punishment." We included a code for positive views for greater detail. These categories reflect what Lakoff and Johnson [3] describe as the ontology (i.e., the nature of being) and the orientation (pertaining to affect) of a metaphor. The expressions were also coded for the four dominant attributions: ability, effort, task difficulty and luck.

The participants were involved in the process of coding to ensure the interpretations of the expressions were as aligned as possible with their intended meaning. This was done to elevate the participants' voices and do research with them, not on them [9]. We sat with individual participants and outlined the literature guiding the study and our proposed analysis. Together we coded the transcripts for the metaphor's ontologies, affective orientation and the factors the participants attribute their success to. Throughout the process, participants were asked to provide suggestions for modifications to the coding scheme.

\section{FINDINGS}

Here we present the participants' responses to the metaphor prompt, summarize the excerpts in terms of the target domain, and highlight themes within their metaphors. We then proceed to a discussion of the insights that emerged from exploring the metaphor characteristics and comparing the participants' metaphors.

\section{A. Participant metaphors}

\section{Samaria}

Samaria prefaces that she wants to explain how success in physics is knowing more things, but not knowing all of them. After a brief pause for thought, she says:

$S: \quad$ [success in physics is like] ...it's like dragging a pail and getting some water out of a wishing- like a well. But like, but just like using like a cup. You know like a standard cup and just using that to get a little bit of water and you just keep trying to get more water and trying to get all the water from the well but you can't obviously cause you're just using the cup.

In summary, Samaria emphasizes how success in physics to her is a continuous process of gaining knowledge. Mapping the target domain to the source domain, the metaphor presents knowledge as water, the ability to gain knowledge in limited amounts as a standard cup and the continuous process of gaining knowledge without ever knowing everything as trying to get all the water but not being able to.

The ontology of Samaria's metaphor is centered around the process of success in physics (you just keep trying to get more) as well as the product (getting some water). However, she makes it clear that while the goal is to get all the water, it is unattainable. For Samaria, the process is just as important, if not more so, than the product. The 
orientation of Samaria's metaphors was difficult to determine. Upon initial inspection, mentions of getting water were tentatively interpreted as positive; however, when discussing the coding with Samaria, she clarified that the knowledge one collects may be positive, negative, or neither. In her words, "some days you might love getting the water and some days you might not." The researcher and Samaria determined that none of her metaphors had an orientation. In her metaphor, Samaria makes hints of attributing success in physics to the effort of dragging the pail/cup.

\section{Renae}

Responding to the same prompt, Renae provided the following metaphor:

R: So, success in physics in grad school feels like running into a door, knowing that at the end the door will be opened for you, but you have to keep running into it. (laughs).

Int: How fast are you running?

$R$ : Well see, depends on like, how badly you want to get through that door (laughs). Like, the faster and harder you run into the door, the sooner it might open for you. (laughs).

Int: It's gonna hurt (laughs).

R: Yeah, it's, it's gonna hurt but, at the end, and at the end it will open because your professors will be like 'Oh, obviously you've run into this enough times now, so you can pass'.

Int: And at what point do you know the door's open?

$R$ : When you fall through, and you're like 'Uh, what? There are things, I'm not running into it anymore?' I don't really know because I haven't actually gone through the open door yet (laughs). I'm still running into the door.

Int: What do you think it's gonna look like in your physics career when the door opens?

R: Um, I'm hoping that it's not just a room full of more doors and I get to choose which one I get to run into next.

Int: Like do your own research and stuff?

R: Yeah, I'm hoping it's just like, I get outside and I can just like go climb a tree or fly a kite.

Renae emphasizes how success in physics as a graduate student is about working continuously until she is provided the opportunity to proceed in a career of her choosing. Her metaphor starts by describing working for a degree in physics as running into a door. She portrays her professor allowing her to continue by expressing that the door will be opened for her. Renae also notes that depending on effort (the faster and harder you run into the door), her advisor will allow her to proceed at an earlier time. She then describes a path of her own choosing by mentioning the possible doors to choose from and going outside to enjoy carefree activities.

Renae uses metaphors that accentuate the process ontology of success in physics (you have to keep running into it). There are products associated with the process of running into the door (at the end the door will be opened), but they relate to not continuing in the current process. In her metaphor, Renae conveyed negative emotions when she talked about running into the door. For her, the metaphors with a process ontology were all negative. Many of the products identified were related to this negative view of the process, since they involved no longer running into the door (When you fall through, and you're like... I'm not running into it anymore?). Although Renae recognizes the adversity, she hints at attributing success to the effort that will lead to success in physics (depends on like, how badly you want to get through that door (laughs). Like, the faster and harder you run into the door, the sooner it might open.)

\section{B. Metaphor characteristics}

\section{Ontology}

We initially thought the ontology of the overarching metaphors would be coded as either process or product (in line with Paulson and Armstrong's coding) [5]. However, we found that method was inadequate, since the metaphors here demonstrated a relationship of the process leading up to the product. In Samaria and Renae's metaphors, they begin their expressions with mentions of the process (like dragging a pail/like running into a door) and then follow with the product (and getting some water/knowing it will be opened). We observed this relationship between the process and product throughout these metaphors and those in the larger sample. Thus, we coded the individual metaphorical expressions as either process, product or both. This nuance suggests that the ontology of success in physics is more complex than that of other concepts such as reading.

\section{Affective Orientation}

Coding the affective orientation of the metaphors allowed for the coding of explicit mentions of emotions or emotionally charged context (like running into a door) but not others with more ambiguous contexts (like dragging $a$ pail). It was particularly difficult to assess the orientation of the metaphors provided by Samaria. After discussing with her, we concluded that some metaphors will not have a clear affective orientation.

\section{Attributions}

Effort was the factor that Samaria and Renae attributed their success to most often. This may be an artifact of both of their metaphors having an emphasis on the process nature of success in physics. Samaria explained during the coding that she values her effort since she has had to be independent due to living with different caretakers throughout her life. 
Renae's emphasis on effort comes from her understanding that as long as she demonstrates her hard work to her advisor, her advisor will eventually allow her to continue. This attribution to effort may be a positive influence in her persistence in physics, since attributions to controllable causes, such as effort, may increase the likelihood of continued engagement in a task [7].

We emergently identified other factors that participants attributed to success that were not encompassed by ability, effort, task difficulty and luck, specifically an external person playing a role in success. Renae exposed this in her first sentence: "So, success is physics in grad school feels like running into a door, knowing that at the end the door will be opened for you..." This statement would be much different if it were instead, "at the end you will open the door." Renae makes it explicit that although she has control over the running that she must do, someone else, her professor is responsible for opening the door for her. The role of her professor pertains to an external cause that she has some influence over.

\section{IMPLICATIONS}

As researchers and educators, we have traditionally seen student success in physics as obtaining products such as high grades and degrees. This study revealed more nuanced perceptions of success in physics leading to the following implications.

Focusing on the products of success in physics neglects the perspectives of those who value the process. Samaria teaches us that, for her, success in physics is more than a product; it is also about the process of seeking knowledge. With this in mind, educators should create opportunities to recognize and appreciate their students' involvement in the processes of physics. Creating these opportunities has been demonstrated to positively shift students' sense of recognition through implicit recognition strategies, such as assigning tasks that are adequately demanding, followed by explicit recognition, such as verbal affirmations [12]. Implementing such recognition strategies can ultimately lead to the development of students' physics identities.
The metaphor presented by Renae also highlights the need to set clear expectations between educators and advisors and their students. As Renae works on her graduate coursework and research, she depicts not making any progress and not knowing when progress will be made. We also need to understand the difficulties (doors) students encounter in order to help the students overcome them (open those doors). We encourage educators and advisors to establish contracts with their students to explicate each other's goals and expectations and set up regular meetings to discuss the struggles and uncertainties students are facing.

From Renae's description of running into doors, we also recognize that success in physics is not exclusively a positive concept. As researchers, this raises a red flag since we often frame our explorations with the assumption that success in physics is a positive outcome. Recent studies problematize these sorts of explorations by considering that the resiliency of Black women in STEM is coupled with socio-emotional demands [13]. They revealed that attaining traditional aspects of success can lead to serious mental, emotional and physical complications. Therefore, it is necessary to continue explorations into success with the recognition that success is a concept that encompasses both positive and negative consequences. Recognizing the complexity of success also allows for explorations into the factors that lead to different perspectives of success. Along with a necessity for ongoing exploration, the ambiguity of success suggests that researchers should make it common practice to operationalize what they mean when they discuss success in the context of physics.

In conclusion, success in physics remains a puzzle that requires further exploration. However, we've revealed aspects of its ontology and troubling perspectives associated with it by using working with our participants to conduct metaphor analysis.

\section{ACKNOWLEDGEMENTS}

This material is based upon work supported by the National Science Foundation Graduate Research Fellowship under Grant No. 1649522. Thank you to Erin Scanlon for feedback.
[1] C. Hill, et al., Why so Few? Women in Science, Technology, Engineering, and Mathematics (AAUW, Washington, D.C, 2010).

[2] R. S. Barthelemy, et al., Phys. Rev. Phys. Ed. Res. 12, 020119 (2016).

[3] G. Lakoff and M. Johnson, Metaphors We Live By (University of Chicago Press, Chicago, 2003).

[4] M. C. M. de Guerrero and O. S. Villamil, Lang. Teach. Res. 6, 95 (2002).

[5] E. J. Paulson and S. L. Armstrong, J. Adolesc. Adult Lit. 54, 494 (2011).

[6] R. E. Scherr and P. R. L. Heron, in Physics Education Research Conference Proceedings, (2016), pp. 316319.
[7] B. Weiner, Psychol. Rev. 92, 548 (1985).

[8] S. Harding, in Handbook of Feminist Research: Theory and Praxis (SAGE Publications, Inc., 2455 Teller

Road, Thousand Oaks California, 2012), pp. 46-64.

[9] L. Harvey, International Journal of Research \& Method in Education 38, 23 (2015)

[10] E. Chase, Qual. Rep. 22, 2689 (2017)

[11] E. J. Paulson and C. K. Theado, Classroom Discourse 6, 1 (2015).

[12] J. Wang and Z. Hazari, Phys. Rev. Phys. Ed. Res. 14, 020111 (2015).

[13] E. O. McGee and L. Bentley, Cogn. Instr. 35, 265 (2017). 